\title{
Changes in pressing for intracranial stimulation (ICS) after prolonged ICS
}

\author{
BRUCE M. BECKER and LARRY D. REID \\ Bradley University, Peoria, Illinois 61606
}

\begin{abstract}
Rats, fixed with two chronically indwelling electrodes, pressed daily for fixed intensities of ICS while press rates were tabulated. As daily testing for press rates continued, there were sessions in which 20 -sec trains of low-intensity $(20 \mu \mathrm{A}$ or less of $60-\mathrm{Hz}$ sine waves) were imposed at some electrode sites. These trains of ICS led to reduced pressing rates for the fixed ICS, but $20 \mathrm{sec}$ of intermittent ICS (.25 sec on, $.25 \mathrm{sec}$ off) or zero ICS did not. It was concluded that $20 \mathrm{sec}$ ICS of low intensity, an ICS frequently used in studies of motivation, reduced the capacity of ICS to sustain pressing, probably by disrupting the tissue of ICS.
\end{abstract}

A number of procedures involve programming durations of intracranial stimulation (ICS) of $20 \mathrm{sec}$ or greater. Using these prolonged trains of ICS, Amit, Stern, and Wise (1970), as well as others following their lead, have demonstrated that daily hypothalamic ICS can lead to increased voluntary consumption of ethanol solutions. The typical procedure is to program $20 \mathrm{sec}$ trains of ICS $(60-\mathrm{Hz}$ sine waves of about $30 \mu \mathrm{A}$ ), with intervals between trains of $20 \mathrm{sec}$, for $30 \mathrm{~min}$ a day. In a rat's home cage, an ethanol solution (typically $20 \%$ ethanol, v/v) and tap water are presented while amounts consumed during day-long periods are tabulated. Rats that received ICS consumed considerable quantities of ethanol (sometimes taking the ethanol solutions almost exclusively) and furthermore maintained high levels of consumption beyond the days during which ICS was programmed.

The finding that hypothalamic ICS can lead to increased consumption of ethanol is interesting and is, in part, the impetus for development of new theory and, in turn, for deriving new treatment practices for alcoholism (Amit \& Sutherland, 1975, 1976; Amit, Levitan, \& Sutherland, Note 1). The finding that ICS leads to heightened ethanol consumption, however, has not been found by everyone (e.g., Martin \& Myers, 1972), suggesting that subtle procedural difference between studies might account for differences in outcomes. Some of the potentially relevant variables have been studied and certain parametric manipulations of germane variables have been accomplished (Wise \& James, 1974). The interpretation of the finding of enhanced ethanol consumption with ICS will, of course, vary depending on the circumstances under which the phenomenon is achieved.

This research was supported by Grant DA 01049, National Institute of Drug Abuse, DHEW, and by a grant from the Distilled Spirits Council of the United States.
One potentially relevant variable that has not been inspected is the possibility that the ICS used in these studies may be disrupting the tissue at the electrode tip. Recently collected data (Magnuson, Tadeusik, \& Reid, 1976; Terando, Mirza, Zipnick, Overmier, Rossi, \& Reid, Note 2) lead to the suggestion that intensities of ICS used in many studies of intracranial selfstimulation are sufficiently intense to disrupt the tissue as indexed by downward shifts in pressing for low-intensity ICS. The purpose of this study was to determine if the ICS used in studies following Amit et al. (1970) is of sufficient magnitude to reduce pressing maintained by low-intensity ICS.

\section{METHOD}

\section{Subjects}

Seven albino (Holtzman, Madison) adult male rats weighing about $350 \mathrm{~g}$ were each fixed, using standard procedures, with two chronically indwelling bipolar electrodes. Each bipolar electrode (Plastic Products) was two strands of platinum wire (diameter of a wire $=.25 \mathrm{~mm}$ ) insulated with Teflon except at the cross section of the tips. The strands were separated from one another only by their insulation. Electrode tips were placed so that ICS by way of each electrode would activate the medial forebrain bundle of the lateral hypothalamus, sites of positive intracranial reinforcement. With bregma and lambda at the same horizontal plane, the stereotaxic coordinates for one electrode were $3.0 \mathrm{~mm}$ posterior to bregma, $1.6 \mathrm{~mm}$ to the right of the rat's midline, and $8.2 \mathrm{~mm}$ ventral to the skull's surface. Coordinates for the other electrode were $3.5 \mathrm{~mm}$ posterior to bregma, $1.6 \mathrm{~mm}$ to the left, and $8.2 \mathrm{~mm}$ ventral. From time of surgery until completion of procedures, rats were housed individually, with food and water always available.

\footnotetext{
Apparatus

The apparatus was a Plexiglas chamber, $30 \times 24 \times 35 \mathrm{~cm}$, housed in a lighted, sound-attenuating box. Extending through one wall of the chamber was a lever, the depression of which yielded a single train of ICS. ICS was $60-\mathrm{Hz}$ sine waves of $0.25 \mathrm{sec}$ of selected intensities but never greater than $40 \mu \mathrm{A}$. ICS was delivered by way of light flexible leads connected to a commutator allowing unhampered movement. Number of presses were recorded automatically at the end of 2-min periods. Procedures involving prolonged ICS were conducted in chambers similar to those of testing, although no lever was present.
} 


\section{Procedure}

Subsequent to recovery from surgery (at least 5 days), rats were allowed to press to verify that ICS to each site would sustain leverpressing. During initial testing, intensity of ICS was varied to select three intensities. For each rat, and each site of ICS, there was a low intensity (limits of range across all electrode sites were 3 to $20 \mu \mathrm{A}, \mathrm{rms}$ ), a medium intensity (ranging from 8 to $24 \mu \mathrm{A}$ ), and a high intensity (from 10 to $40 \mu \mathrm{A}$ ). The low intensities were just intense enough to maintain steady pressing; the high intensity was that intensity which sustained high rates of pressing; and the medium intensity was about midway between the other two.

After selection of intensities of ICS, the rats were given opportunities to press daily. With an intensity of ICS, each opportunity to press lasted 2 min and was preceded by at least 10 ratinitiated presses or 10 experimenter-initiated ICS given while shaping the rat to the lever. The order of testing by way of each electrode was always first for low ICS and last for high ICS. Flanagan (1975) demonstrated that it made little difference in scores from similar tests when order of testing was varied. The sequence of tests was always begun with the anterior site. Consequently, a daily test was a series of opportunities to press for ICS, first for low, medium, and high ICS of the anterior electrode site, followed by low, medium, and high ICS of the posterior electrode site.

After rates of pressing stabilized (at least 18 days of consecutive daily sessions, during which time ICS intensities were varied slightly until rats pressed similarly at both sites), testing continued for 3 days, the tests of baseline. Intensities of ICS for the balance of the testing procedures, i.e., the procedures of selfstimulation, were those of baseline. On the day following baseline, different regimens of prolonged ICS were introduced to randomly selected electrode sites while tests for pressing continued as before. Prolonged ICS procedures followed a regular daily test session at the selected standard intensities of baseline. Consequently, tests for any effects of the prolonged ICS were conducted $24 \mathrm{~h}$ following the imposition of prolonged ICS procedures and immediately prior to the next session of imposed ICS.

Three subjects were given $20 \mathrm{sec}$ of ICS followed by $20 \mathrm{sec}$ of no ICS for $30 \mathrm{~min}$, or 45 trains of ICS of $20 \mathrm{sec}$. Two subjects were given long trains of ICS by way of the anterior electrode, while the other received the ICS by way of the posterior elec- trode (the experimental site for those subjects). Two other rats received $20 \mathrm{sec}$ of intermittent ICS immediately followed by $20 \mathrm{sec}$ of no ICS for $30 \mathrm{~min}$. The intermittent trains were $0.25 \mathrm{sec}$ of ICS, with $0.25 \mathrm{sec}$ of no ICS for the $20 \mathrm{sec}$. One rat received the prolonged but intermittent ICS by way of the anterior electrode, the other by way of the posterior electrode (the experimental sites). The remaining two subjects received zero ICS for the $30 \mathrm{~min}$, i.e., all the procedures were the same as those of constant trains, except the intensity of "imposed" ICS was zero. The lead was attached to the anterior electrodes for one subject and the posterior for the other (their "experimental sites"). The sites not designated experimental sites were labeled "control sites," and there was one experimental and one control site for each rat. There was no imposed ICS at any control site.

Intensities of the prolonged ICS began with ICS of $5 \mu \mathrm{A}$ (except for the no-ICS experimental sites, which always received $0-\mu \mathrm{A}$ ICS) and increased by $5 \mu \mathrm{A}$ daily until the intensity of $20 \mu \mathrm{A}$ was reached. Sessions of prolonged ICS at $20 \mu \mathrm{A}$ continued for 10 days. The $20-\mu \mathrm{A}$ intensity was chosen because it was the mean of the median intensities used by Amit et al., (1970) and was similar to other studies following Amit et al.

After the last day of testing, subjects were sacrificed under large doses of anesthesia followed by intracardial perfusions of saline and Formalin. Eventually, 60-micron frozen slices of each brain were inspected to determine the sites of the electrode tips. Slices were treated as photographic negatives, and the resulting images were enlarged and photographically recorded as aids in inspecting the sites of ICS (Guzman-Flores, Alcarez, \& Fernandez, 1968).

To summarize, each rat was tested daily for self-stimulation rates at each electrode site, with three standard intensities of ICS across days of the procedure. On some days, just after selfstimulation tests, one randomly chosen electrode site was programmed in each rat with (a) 20-sec trains of ICS, (b) $20 \mathrm{sec}$ of intermittent ICS (0.25 sec on and $0.25 \mathrm{sec}$ off $)$, or (c) no ICS but electrode attachment. Consequently, one electrode site in each rat was a control allowing an assessment of the effects of imposing ICS and both sites in two rats were also controls. The effects of imposing the ICS can be discerned, therefore, by comparing responsiveness to standard intensities of ICS across the two sites in a single rat and by comparing responsiveness between rats of imposed ICS and rats of zero ICS.

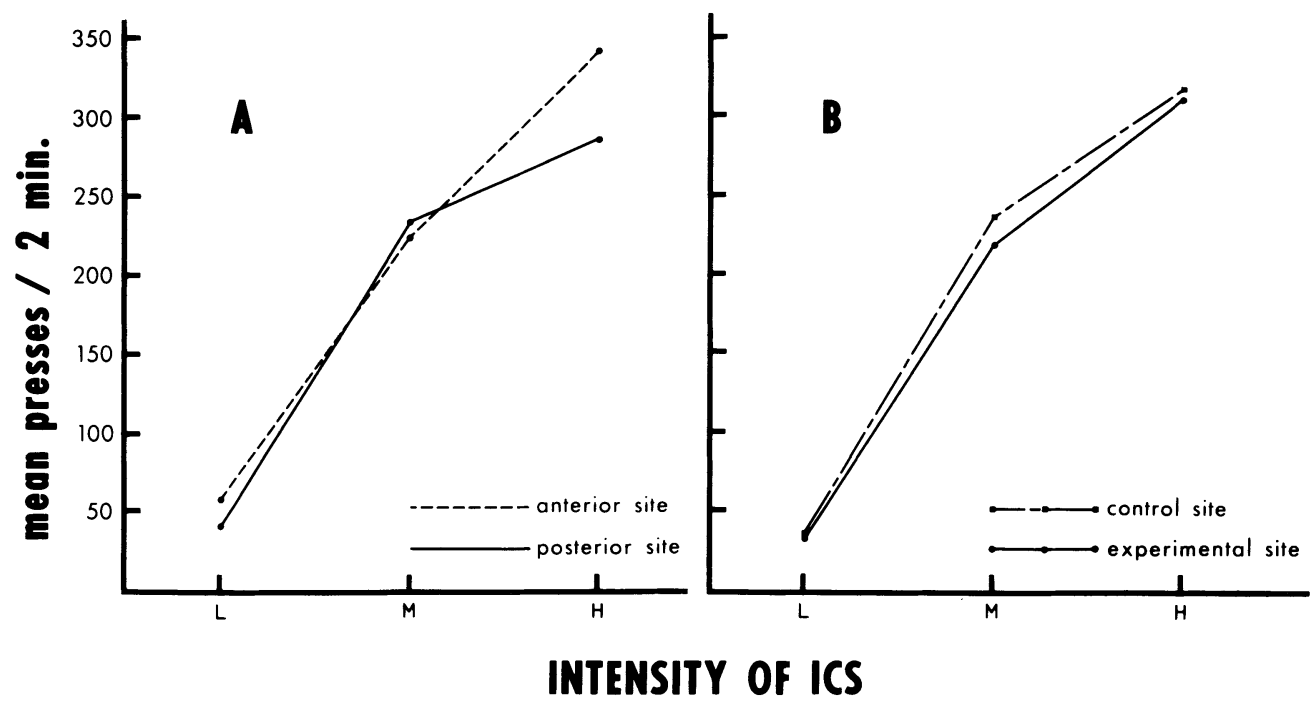

Figure 1. Mean pressing rates of the rats prior to introduction of prolonged ICS procedures. 


\section{RESULTS}

Preliminary procedures had as goals that pressing would be similar for both posterior and anterior ICS and that there would be differential pressing for the low, medium, and high ICS. Panel A of Figure 1 shows that these goals were achieved by depicting mean pressing rates for low, medium, and high ICS for both anterior and posterior sites. A 2 by 3 analysis of variance (ANOVA) was done with factors associated with anterior-posterior sites and intensities using mean press rates across days of baseline as the raw data. That ANOVA yields an $F$ for the factor of Site of $<1$, an $F(2,12)$ for the factor of Intensity $=57.10, \mathrm{p}<.01$, and an $F(2,12)$ for the interaction $=1.77, \mathrm{p}<.25$. Panel $\mathrm{B}$ of Figure 1 shows that pressing rates were similar for ICS at the experimental and control sites prior to the introduction of the procedures of imposed ICS. The ANOVA of Panel B's data yielded an F for the factor of Site of $<1$, an $F(2,12)$ for the factor of Intensity = $57.17, \mathrm{p}<.01$, and $\mathrm{F}$ for the interaction of $<1$.

Data showing the effects on pressing of imposing prolonged trains of ICS are summarized in Figure 2. The data points of Figure 2 are mean ratios. A ratio was computed by dividing a test score by its corresponding baseline score (mean of tests across 3 days of testing just prior to the procedures of imposing prolonged ICS, the scores germane to Figure 1). A ratio greater than 1.00 indicates an increase in pressing during test procedures over those of baseline and a ratio less than 1.00 indicates a decrease. These ratio scores also have the effect of reducing variance due to the factor of intensity. Consequently, analyses of ratio scores will not necessarily yield reliable intensity effects as with the scores of Figure 1.

Panel A of Figure 2 presents mean ratios following imposition of constant train of ICS of 5, 10, and $15 \mu \mathrm{A}$ and mean ratios across the first and second 5 days following imposition of $20 \mu \mathrm{A}$. It can be seen that pressing on the side of imposed ICS was decreased compared to pressing on the other side. Student $t$ tests comparing each pair of data points of the tests following imposition of 5,10 , or $15 \mu \mathrm{A}$ provide no basis for concluding that performance for ICS of the two sites differed except for the tests with low intensities following 10 and $15 \mu \mathrm{A}, \mathrm{t}(4)$ for the $10 \mu \mathrm{A}$-test $=3.78, \mathrm{p}<.01, \mathrm{t}(4)$ for the $15 \mu \mathrm{A}$-test $=2.42, \mathrm{p}<.05$. A 2 by 10 by 3 ANOVA having repeated measures with factors associated with experimental-control sites (imposed ICS-no imposed ICS), the 10 daily tests concurrent with imposition of $20 \mu \mathrm{A}$, and three intensities of ICS using ratios as the raw data was done. That ANOVA yields an $F(1,4)$ for the factor of Site $=16.39, p<.05$; an $F(2,8)$ for the interaction of Site by Intensity $=13.87$, $\mathrm{p}<.01$; an $\mathrm{F}(9,36)$ for the factor of Daily Tests = $1.95, \mathrm{p}<.10$. All other factors yielded Fs with associated $p$ values $>.20$. Student $t$ tests were used to compare mean ratios of the two sites at each intensity. These tests indicated that pressing was reliably different for the low ICS, $t(4)=4.12$, $\mathrm{p}<.01$, and for the medium ICS, $\mathrm{t}(4)=3.55$,

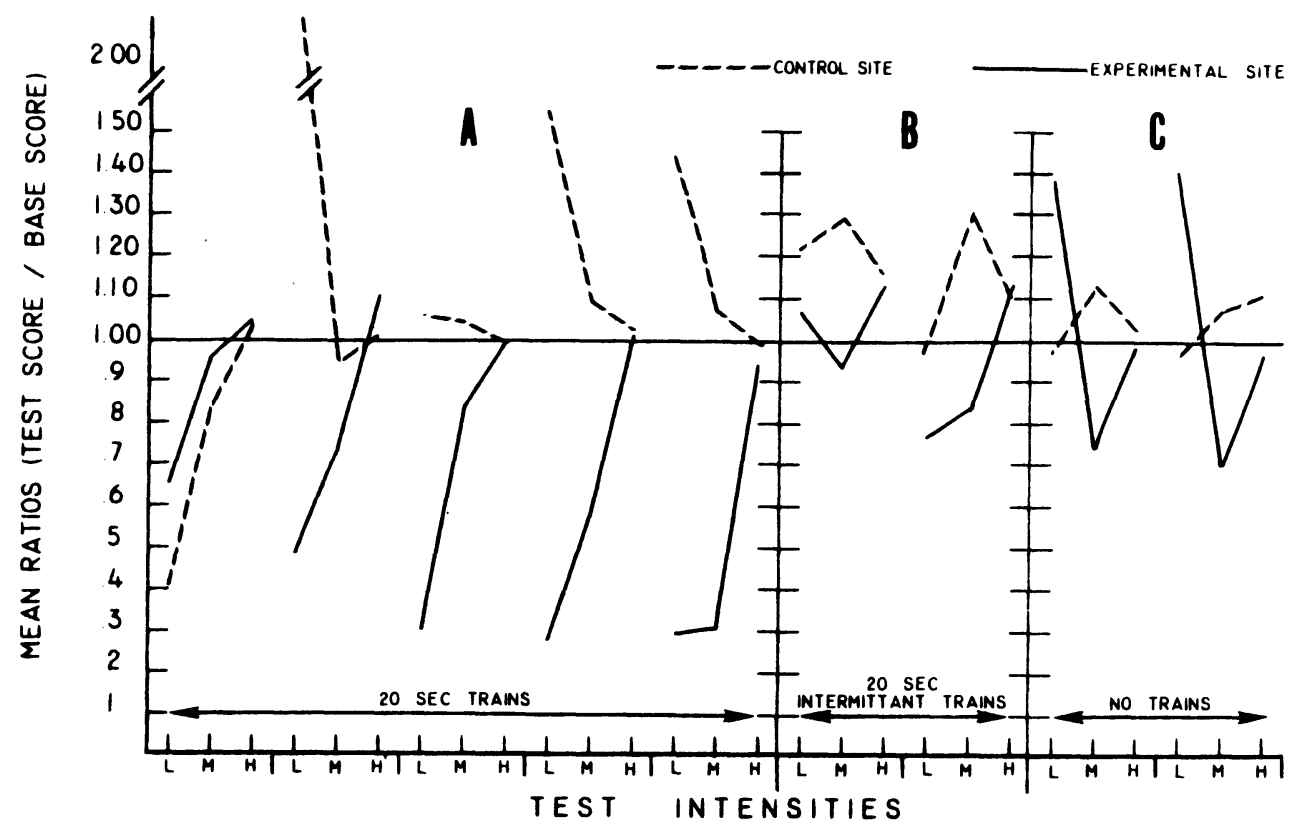

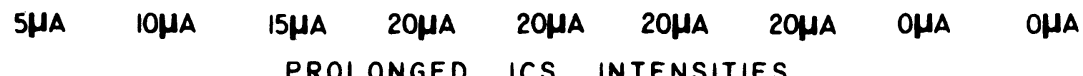

Figure 2. Mean ratio scores (means of scores of test sessions/scores of comparable baseline) following imposition of constant, intermittent, or zero intensity trains of ICS. 
$\mathrm{p}<.05$, but not for the high ICS. To summarize, imposing 20 -sec trains of 10 or $15 \mu \mathrm{A}$ reliably reduces pressing for ICS just above threshold for self-stimulation, and imposing $20 \mathrm{sec}$ trains of $20 \mu \mathrm{A}$ reliably reduces pressing for low and medium ICS but does not reliably modify pressing rates for high ICS (20 $\mu \mathrm{A}$ in these subjects).

Panel B of Figure 2 presents a summary of the data relevant to assessing the effects of the prolonged but intermittent trains of ICS. Data of 5, 10, and $15 \mu \mathrm{A}$ of intermittently imposed ICS are not presented in Figure 2, since these intensities, like the higher intensity of $20 \mu \mathrm{A}$, did not disrupt pressing. An ANOVA (a 2 by 10 by 3 factorial having repeated measures) of the data from which Panel B was derived provides no support for the hypothesis that the prolonged but intermittent ICS modified responsiveness to fixed ICS ( $\mathrm{ps}$ for all factors $>.25$ ).

Panel $\mathrm{C}$ of Figure 2 presents data of the two rats that had no ICS imposed. An ANOVA (a 2 by 10 by 3 factorial having repeated measures) of the data of Panel C indicates no reliable effect associated with the imposition of $0-\mu \mathrm{A}$ ICS (ps for all factors $>.20$ ). As can be seen, the data of Panel B are similar to that of Panel C, i.e., responsiveness following intermittent ICS is similar to that of no ICS. The data of imposed ICS procedures of Panel A, however, are quite different from those of Panels $B$ and $C$, i.e., responsiveness for fixed ICS following prolonged trains reduced pressing, whereas pressing had a tendency to increase in every other instance. It did not seem to matter whether the prolonged ICS was imposed through the anterior or posterior sites.

Almost all electrode sites were within the lateral hypothalamus, and the ICS probably activated the medial forebrain bundle (Figure 3). In one rat of constant trains of ICS, the sites of stimulation were dorsal to the median forebrain bundle in Forel's fields. All sites were lateral to the fornix columns and medial to the crus cerebri. The sites ranged from the anterior to the posterior lateral hypothalamus. From inspection of the enlarged (X10) photographs, it was impossible to discern which sites had sustained the ICS that disrupted pressing for lowintensity ICS.

\section{DISCUSSION}

It is typical for rats pressing daily for ICS to gradually increase their pressing across days of testing, especially for lower intensities (Bush, Bush, Miller, \& Reid, 1976). Consequently, it is not surprising to see, in general, increased pressing for ICS to control sites. The fact that pressing rates decreased with $20-\mathrm{sec}$ trains of $10 \mu \mathrm{A}$ or greater, and that rates would be expected to increase gradually without imposition of the trains, points to a relatively dramatic difference depending on whether or not prolonged

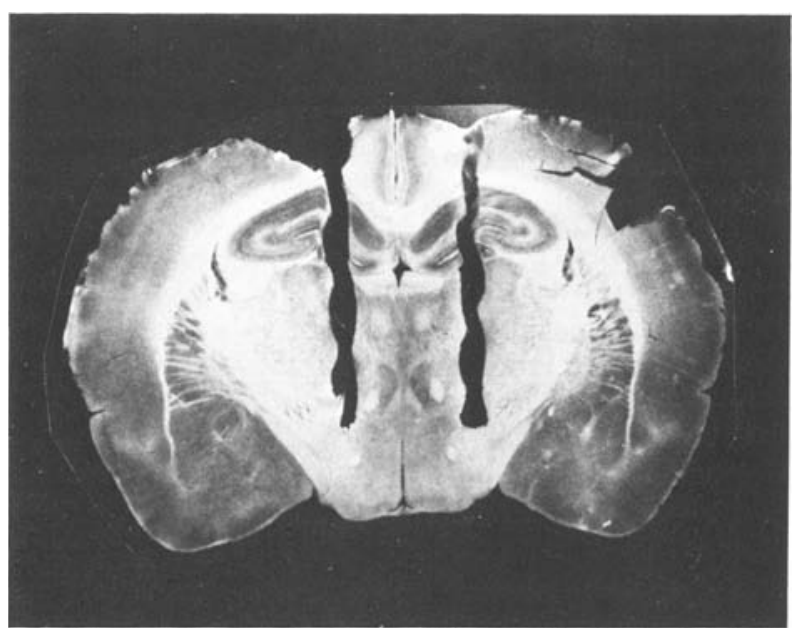

Figure 3. Photograph showing the two electrode sites of one rat. These two sites are somewhat closer to one another than those of other subjects. The site on the right sustained the constant trains of ICS.

ICS is imposed. Although it is clear that there is a difference, nothing about our procedures specifies the details of that difference. The prolonged ICS may have destroyed neurons, may have altered surrounds of neurons, such as blood flow or glial cells, or may have left cells fundamentally intact but modified their functions.

The fact that 20-sec trains of ICS can modify the tissue of ICS opens the question of what events might have induced ethanol consumption in studies following Amit et al., (1970). Results lead to the possibility that shifts in kinds of stimulus-bound behaviors, as demonstrated by Valenstein, Cox, and Kakolewski (1969) following ICS similar to that used in this study, are due to modifications of the tissue at the electrode tip and therefore do not represent an inherent plasticity in the organization of the hypothalamus. Many procedures (e.g., those reviewed by Hoebel, 1974) involve programming prolonged trains of ICS, and it would seem prudent to take into account the strong possibility that at least the initial impositions of prolonged trains may not be merely activating the tissue.

If the goal of imposing ICS is to produce high levels of activity at the electrode tip without producing apparent damage, then it appears that the intermittent ICS of $.25 \mathrm{sec}$ on and $.25 \mathrm{sec}$ off is the ICS to use. This intermittent ICS should produce high levels of sustained neural activity without, apparently, the complications of disrupting the stimulated tissue.

\section{REFERENCE NOTES}

1. Amit, Z., Levitan, D. E., \& Sutherland, E. A. Development of a treatment model for alcoholics. Paper presented at the Eleventh 
Annual Conference of the Canadian Foundation on Alcohol and Drug Dependencies, Toronto, Canada, June 1976.

2. Terando, L., Mirza, N., Zipnick, J., Overmier, M., Rossi, N., \& Reid, L. Addictive agents and intracranial stimulation (ICS): Daily morphine, self-stimulation, and parameters of ICS. In preparation.

\section{REFERENCES}

Amit, Z., Stern, M. H., \& Wise, R. A. Alcohol preference in the laboratory rat induced by hypothalamic stimulation. Psychopharmacologia (Berlin), 1970, 17, 367-377.

Amit, Z., \& SutherLAND, E. A. The relevance of recent animal studies for the development of treatment procedures for alcoholics. Drug and Alcohol Dependence, 1975/76, 1, 3-13.

Bush, H. D., Bush, M. F., Miller, M. A., \& ReID, L. D. Addictive agents and intracranial stimulation: Daily morphine and lateral hypothalamic self-stimulation. Physiological Psychology, 1976, 4, 79-85.

FLANAGEN, R. W. Dose-related effects of ethanol on intracranially reinforced response. Unpublished doctoral dissertation, Indiana University, 1975.
Guzman-Flores, C., Alcarez, M., \& Fernandez, A. Rapid procedure to localize electrodes in experimental neurophysiology. Boletin del Institute de Estudios Medicos y Biologicos, Mexico, 1968, 16, 29-31:

HOEBEL, B. G. Brain reward and aversion systems in the control of feeding and sexual behavior. In J. K. Cole \& T. B. Sondergger (Eds.), Nebraska symposium on motivation (Vol. 22). Lincoln: University of Nebraska Press, 1974.

Magnuson, D. J., Tadeusik, C. J., \& Reid, L. D. Addictive agents and intracranial stimulation: Self-stimulation under morphine, amphetamine, and chlorpromazine. Bulletin of the Psychonomic Society, 1976, 8, 459-462.

MARTIN, G. E., \& MYERs, R. D. Ethanol ingestion in the rat induced by rewarding brain stimulation. Physiology and Behavior, 1972, 8, 1151-1160.

Valenstein, E. S., Cox, V. C., \& Kakolewski, J. W. The hypothalamus and motivated behavior. In J. T. Tapp (Ed.), Reinforcement and behavior. New York: Academic Press, 1969. WISE, R. A., \& JAmEs, L. Rat ethanol intake: Suppression by intracranial surgery and facilitation by intracranial stimulation. Psychopharmacologia (Berlin), 1974, 37, 179-184.

(Received for publication August 12, 1976; revision accepted November 4,1976 .) 